\title{
Synthesis and biodegradation of three poly(alkylene succinate)s: mathematical modelling of the esterification reaction and the enzymatic hydrolysis
}

\author{
D. S. Achilias \& D. N. Bikiaris \\ Laboratory of Organic Chemical Technology, Department of Chemistry, \\ Aristotle University of Thessaloniki, Thessaloniki, Greece
}

\begin{abstract}
Synthesis of three biodegradable aliphatic polyesters, namely poly(ethylene succinate), poly(propylene succinate) and poly(butylene succinate), is presented using the appropriate diols and succinic acid. A theoretical mathematical model for the esterification reaction is developed and applied successfully in the simulation of all experimental data. Different glycols do not influence the average degree of polymerization of the oligomers produced much, even though they slightly affect esterification rates in the order $\mathrm{BG}>\mathrm{PG}>\mathrm{EG}$. In contrast, these values are affected by the amount of catalyst, with a larger catalyst molar ratio giving a polymer with a larger than average molecular weight. Biodegradability studies of the polyesters included enzymatic hydrolysis for several days using Rhizopus delemar lipase at $\mathrm{pH} 7.2$ and $30^{\circ} \mathrm{C}$. The biodegradation rates of the polymers decreased following the order $\mathrm{PPSu}>\mathrm{PESu} \geq \mathrm{PBSu}$ and it was attributed to the lower crystallinity of $\mathrm{PPSu}$ compared to other polyesters, rather than to differences in chemical structure. Finally, a simple theoretical kinetic model was developed and Michaelis-Menten parameters were estimated.
\end{abstract}

Keywords: biodegradable polymers, enzymatic hydrolysis, aliphatic polyesters, mathematical modelling.

\section{Introduction}

Continuous environmental surcharge due to domestic and industrial waste consists of one of the major problems of growth nowadays. Plastic waste 
materials represent a high percentage of domestic waste and their amount continues to increase, despite some increasing attempts to reduce, reuse, recycle and recover. This is mainly due to their wide application in the manufacture of packaging for the food industry as well as in other goods of daily life. Although most plastic wastes are not toxic and do not create a direct hazard to the environment, they are seen as noxious materials due to their substantial fraction by volume in the waste stream and their high resistance to the atmospheric and biological agents. Therefore, the development and application of biodegradable polymers, instead of those traditionally used, has received a great attention from the viewpoint of environmental protection and resource recycle. Among synthetic polymers, aliphatic polyesters combine the features of biodegradability, biocompatibility and physical or chemical properties, comparable with some of the extensively used polymers like polyethylene (LDPE), polypropylene (PP), etc.

In the present investigation, three aliphatic polyesters, namely poly(ethylene succinate), poly(propylene succinate) and poly(butylene succinate) were prepared from succinic acid and ethylene, propylene and butylene glycol, respectively. The advantage of these polyesters is that despite their low melting point they have very high thermal stability, which is familiar to aromatic polyesters like poly(ethylene terephthalate) and poly(butylene terephthalate). The kinetics of the esterification reaction of these polyesters are examined and a detailed mathematical model is developed illustrating the effect of the monomer type together with the catalyst used on the time required to complete the reaction. Furthermore, a comparative biodegradability study is carried out and a theoretical enzymatic hydrolysis kinetic model is introduced.

\section{Experimental}

\subsection{Materials}

Succinic acid (purum $99 \%$ ), ethylene glycol (purum $99 \%$ ) and butylene glycol (purum $99 \%$ ) were purchased from Aldrich Chemical Co. 1,3-Propanediol (CAS Number: 504-63-2, Purity: > 99,7 \%) was kindly supplied by Du Pont de Nemours Co. Tetrabutoxy-titanium (TBT) used as catalyst was analytical grade and purchased from Aldrich Chemical Co. Polyphosphoric acid (PPA) used as heat stabilizer was supplied from Fluka. Rhizopus delemar lipase was purchased from BioChemika. All other materials used were of analytical grade.

\subsection{Synthesis of polyesters}

Synthesis of aliphatic polyesters was performed following the two-stage melt polycondensation method (esterification and polycondensation) in a glass batch reactor (Karayannidis et al. [1]). In brief, the proper amount of succinic acid $(0.55 \mathrm{~mol})$ and appropriate glycols (ethylene glycol, 1,3-propanediol and butylene glycol) in a molar ratio $1 / 1.1$ and the catalyst TBT $\left(3 \times 10^{-4} \mathrm{~mol}\right.$ $\mathrm{TBT} / \mathrm{mol} \mathrm{SA}$ ) were charged into the reaction tube of the polycondensation apparatus. The reaction mixture was heated at $190^{\circ} \mathrm{C}$ under argon atmosphere and stirring at a constant speed (500 rpm) was applied. This first step 
(esterification) is considered to complete after the collection of almost the theoretical amount of $\mathrm{H}_{2} \mathrm{O}$, which was removed from the reaction mixture by distillation and collected in a graduate cylinder. In the case of PPSu two additional catalyst concentrations, namely $1.5 \times 10^{-4}$ and $6 \times 10^{-4} \mathrm{~mol} \mathrm{TBT} / \mathrm{mol} \mathrm{SA}$ were used in order to study the effect of catalyst amount on esterification and polycondensation reactions.

In the second step of polycondensation, PPA was added $\left(5 \times 10^{-4} \mathrm{~mol}\right.$ $\mathrm{PPA} / \mathrm{mol} \mathrm{SA}$ ) and a vacuum (5.0 Pa) was applied slowly over a period time of about $30 \mathrm{~min}$. The temperature was increased to $230^{\circ} \mathrm{C}$ while stirring speed was slowly increased to $720 \mathrm{rpm}$. The polycondensation reaction time was always kept constant at $1 \mathrm{~h}$ and afterwards the polyesters were maintained at room temperature in order to be cooled.

\subsection{Biodegradation studies (enzymatic hydrolysis)}

Polyesters in the form of films with $5 \times 5 \mathrm{~cm}$ in size and approximately $2 \mathrm{~mm}$ thickness, prepared in a hydraulic press, were placed in petries containing phosphate buffer solution ( $\mathrm{pH}$ 7.2) with $1 \mathrm{mg} / \mathrm{mL}$ Rhizopus delemar lipase. The petries were then incubated at $30 \pm 1^{\circ} \mathrm{C}$ in an oven for several days while the media were replaced every 3 days. After a specific period of incubation, the films were removed from the petri, washed with distilled water and weighted until constant weight. The degree of biodegradation was estimated from the mass loss and molecular weight reduction as measured by GPC. In all polyester films intrinsic viscosity, end groups analysis and thermal analysis were performed.

\section{Mathematical modeling of the esterification reaction for the poly(alkylene succinate) synthesis}

\subsection{Reaction scheme}

In this paper the polymer segment approach, which is in the framework of functional group approaches was used (Kang et al. [2]) following our previous article on modeling of the poly(propylene terephthalate) esterification kinetics (Karayannidis et al. [1]). According to this approach, the polymerization reaction is regarded as a reaction between two functional groups. The molecular structure of the components considered in the reaction scheme, is presented in Table 1. The esterification reaction of succinic acid (SA) with three glycols is considered here (i.e. EG ethylene glycol, PG propylene glycol and BG butylene glycol). Five different oligomeric segments are used: tSA, tG, bSA, bG and bDG (the term $\mathrm{t}$ and $\mathrm{b}$ refer to the terminal functional group and bound monomeric repeating unit).

Based on these functional groups, the following mechanism is assumed to represent the kinetics of the esterification process [1].

$$
\begin{array}{r}
\mathrm{SA}+\mathrm{G} \underset{\mathrm{k}_{1}^{\prime}}{\frac{\mathrm{k}_{1}}{\rightleftharpoons}} \mathrm{tSA}+\mathrm{tG}+\mathrm{W} \\
\mathrm{tSA}+\mathrm{G} \underset{\mathrm{k}_{2}^{\prime}}{\stackrel{\mathrm{k}_{2}}{\rightleftharpoons}} \mathrm{bSA}+\mathrm{tG}+\mathrm{W}
\end{array}
$$




$$
\begin{aligned}
& \mathrm{SA}+\mathrm{tG} \underset{\mathrm{k}_{3}^{\prime}}{\stackrel{\mathrm{k}_{3}}{\rightleftharpoons}} \mathrm{tSA}+\mathrm{bG}+\mathrm{W} \\
& \mathrm{tSA}+\mathrm{tG} \underset{\mathrm{k}_{4}^{\prime}}{\stackrel{\mathrm{k}_{4}}{\rightleftharpoons}} \mathrm{bSA}+\mathrm{bG}+\mathrm{W} \\
& \mathrm{tG}+\mathrm{tG} \underset{\mathrm{k}_{5}^{\prime}}{\stackrel{\mathrm{k}_{5}}{\rightleftharpoons}} \mathrm{bG}+\mathrm{G} \\
& \mathrm{tG}+\mathrm{tG} \stackrel{\mathrm{k}_{6}}{\longrightarrow} \mathrm{bDG}+\mathrm{W}
\end{aligned}
$$

Table 1: $\quad$ Molecular structure of components considered.

\begin{tabular}{llc}
\hline Symbol & Description & Molecular Structure \\
\hline SA & Succinic acid & $\mathrm{HOOC}-\mathrm{CH}_{2}-\mathrm{CH}_{2}-\mathrm{COOH}$ \\
$\mathrm{G}$ & ethylene glycol & $\mathrm{HO}-\mathrm{CH}_{2}-\mathrm{CH}_{2}-\mathrm{OH}$ \\
& propylene glycol & $\mathrm{HO}-\mathrm{CH}_{2}-\mathrm{CH}_{2}-\mathrm{CH}_{2}-\mathrm{OH}$ \\
& butylene glycol & $\mathrm{HO}-\mathrm{CH}_{2}-\mathrm{CH}_{2}-\mathrm{CH}_{2}-\mathrm{CH}_{2}-\mathrm{OH}$ \\
W & Water & $\mathrm{H}_{2} \mathrm{O}$ \\
tSA & SA end group & $\mathrm{HOOC}-\mathrm{CH}_{2}-\mathrm{CH}_{2}-\mathrm{CO}-$ \\
tG & EG end group & $\mathrm{HO}-\mathrm{CH}_{2}-\mathrm{CH}_{2}-\mathrm{O}-$ \\
& PG end group & $\mathrm{HO}-\mathrm{CH}_{2}-\mathrm{CH}_{2}-\mathrm{CH}_{2}-\mathrm{O}-$ \\
& BG end group & $\mathrm{HO}-\mathrm{CH}_{2}-\mathrm{CH}_{2}-\mathrm{CH}_{2}-\mathrm{CH} \mathrm{H}_{2}-\mathrm{O}-$ \\
bSA & SA repeating unit & $-\mathrm{OC}_{2}-\mathrm{CH}_{2}-\mathrm{CH}_{2}-\mathrm{CO}-$ \\
bG & EG repeating unit & $-\mathrm{O}_{-}-\mathrm{CH}_{2}-\mathrm{CH}_{2}-\mathrm{O}-$ \\
& PG repeating unit & $-\mathrm{O}_{2}-\mathrm{CH}_{2}-\mathrm{CH}_{2}-\mathrm{CH}_{2}-\mathrm{O}-$ \\
& BG repeating unit & $-\mathrm{O}-\mathrm{CH}_{2}-\mathrm{CH}_{2}-\mathrm{CH}_{2}-\mathrm{CH}_{2}-\mathrm{O}-$ \\
bDG & Diethylene glycol repeat. unit & $-\mathrm{O}_{2}-\mathrm{CH}_{2} \mathrm{CH}_{2}-\mathrm{O}_{-}-\mathrm{CH}_{2} \mathrm{CH}_{2}-\mathrm{O}-$ \\
& Dipropylene glycol rept. unit & $-\mathrm{O}_{2}-\mathrm{CH}_{2} \mathrm{CH}_{2} \mathrm{CH}_{2}-\mathrm{O}_{-}-\mathrm{CH}_{2} \mathrm{CH}_{2} \mathrm{CH}_{2}-\mathrm{O}-$ \\
& Dibutylene glycol rept. unit & $-\mathrm{O}-\mathrm{CH}_{2} \mathrm{CH}_{2} \mathrm{CH}_{2} \mathrm{CH}_{2}-\mathrm{O}-\mathrm{CH}_{2} \mathrm{CH}_{2} \mathrm{CH}_{2} \mathrm{CH}_{2}-\mathrm{O}-$ \\
\hline
\end{tabular}

Reactions 1-4 represent the typical esterification reactions, while reaction 5 is the polycondensation reaction, occurring mainly in the second step of polyester formation. Finally, reaction 6 is a side reaction resulting in di-glycol repeating units, with ether linkages in the oligomeric chain. $k_{i}(i=1,6)$ and $k_{i}^{\prime}(i=1,5)$ represent the kinetic rate constants of the 6 elementary reactions $\left(\mathrm{L} \mathrm{mol}^{-1} \mathrm{~min}^{-1}\right)$.

\subsection{Development of the mathematical model}

In order to develop a mathematical model for the esterification reaction the following assumptions are made: all kinetic rate constants are independent of polymer chain length; all the water produced during the reaction is instantaneously vaporized and removed; all glycol vaporized is totally returned to the reactor. Based then on the reaction mechanism, the reaction rates can be expressed in terms of the different functional groups present in the reactor and the corresponding rate constants [2].

$$
\begin{gathered}
R_{1}=\left\{4 k_{1}(S A)(G)-\left(k_{1} / K_{1}\right)(t S A)(W)\right\} / V^{2} \\
R_{2}=\left\{2 k_{2}(t S A)(G)-2\left(k_{2} / K_{2}\right)(b S A)(W)\right\} / V^{2} \\
R_{3}=\left\{2 k_{3}(S A)(t G)-\left(k_{3} / K_{3}\right)(t S A)(W)\right\} / V^{2}
\end{gathered}
$$




$$
\begin{gathered}
R_{4}=\left\{k_{4}(t S A)(t G)-2\left(k_{4} / K_{4}\right)(b S A)(W)\right\} / V^{2} \\
R_{5}=\left\{k_{5}(t G)(t G)-4\left(k_{5} / K_{5}\right)(b G)(G)\right\} / V^{2} \\
R_{6}=\left\{k_{6}(t G)(t G)\right\} / V^{2}
\end{gathered}
$$

Furthermore the material mole balance equations for a semi-batch reactor can be written as:

$$
\begin{gathered}
\frac{1}{V} \frac{d(S A)}{d t}=-R_{1}-R_{3} \\
\frac{1}{V} \frac{d(G)}{d t}=-R_{1}-R_{2}+R_{5} \\
\frac{d(W)}{d t}=V\left(R_{1}+R_{2}+R_{3}+R_{4}+R_{6}\right)-F_{w}=0 \\
\frac{1}{V} \frac{d(t S A)}{d t}=R_{1}-R_{2}+R_{3}-R_{4} \\
\frac{1}{V} \frac{d(t G)}{d t}=R_{1}+R_{2}-R_{3}-R_{4}-2 R_{5}-2 R_{6} \\
\frac{1}{V} \frac{d(b S A)}{d t}=R_{2}+R_{4} \\
\frac{1}{V} \frac{d(b G)}{d t}=R_{3}+R_{4}+R_{5} \\
\frac{1}{V} \frac{d(b D G)}{d t}=R_{6}
\end{gathered}
$$

In eqn (15), $F_{W}$ is used to represent the flow rate of the water vaporized and removed from the reactor. The total moles of water removed up to time $t, \mathrm{~N}_{\mathrm{W}}$, are:

$$
N_{W}=\int_{0}^{t} F_{W} \cdot d t
$$

Finally, conversion according to the water produced and removed can be calculated according to the following equation:

$$
X_{W}=\frac{N_{W} M W_{W}}{19.8}
$$

Using the polymer segment approach the number average degree of polymerization (NADP) of oligomers produced can be expressed as [2]:

$$
\overline{D P}_{n}=\frac{(t S A)+(b S A)+(t G)+(b G)+(b D G)}{(t S A)+(t G)}
$$

The system of differential eqns (13-20) was integrated by applying the Runge-Kutta $4^{\text {th }}$ order method with varying step size. In order to have the mole number of every component in the reaction mixture as a function of time the kinetic rate constants have to be evaluated. According to the reaction mechanism, 11 rate constants $\left(\mathrm{k}_{\mathrm{i}}, \mathrm{i}=1-6\right.$ and $\left.\mathrm{k}_{\mathrm{i}}^{\prime}, \mathrm{i}=1-5\right)$ should be determined. Since there are not any experimental data in the literature on the polymerization of SA with either EG, PG or BG, all these parameters were estimated according 
to the following assumptions. In PET and poly(propylene terephthalate) polymerization it was assumed that the reactivity of the acid end group on (terephthalic acid) TPA is equivalent to the reactivity on oligomer chain (tTPA), whereas the reactivity of hydroxyl end group on EG was twice of the reactivity on half-esterified EG (tEG) [1,2]. Since in the polymerization of either PG or BG the end groups are identical, we used the same assumption, i.e. $\mathrm{k}_{1}=\mathrm{k}_{2}=2 \mathrm{k}_{3}=$ $2 \mathrm{k}_{4}$. In order to simplify further the calculations it was assumed that $\mathrm{k}_{5}=0$, i.e. that the poly-condensation reaction is not carried out in a great extent during the esterification step. The equilibrium constants $\mathrm{K}_{\mathrm{i}}, \mathrm{i}=1-5$ need not to be evaluated since according to the assumptions made the reverse reaction rates are zero $[(W)=0$ in the liquid phase]. From the above analysis the number of parameters that need to be evaluated is only two, namely $\mathrm{k}_{1}$ and $\mathrm{k}_{6}$.

\section{Results and discussion}

\subsection{Esterification reaction}

The values of the kinetic parameters were calculated for every different system studied from fitting to the experimental data and they are reported in Table 2. The effect of the catalyst molar ratio on the rate of the PPSu esterification reaction is presented in Figure 1. It is obvious that the theoretical model simulates the experimental data very well at all catalyst amounts. As it was also reported for $\mathrm{PBSu}$ [3], an increase in the catalyst concentration leads to increased reaction rates. Furthermore, it was observed that when plotting $\mathrm{k}_{1}$ versus the catalyst concentration in a double logarithmic plot a very good straight line was obtained and the equation thus calculated was: $\mathrm{k}_{1}=10^{-0.91}(\mathrm{~mol} \mathrm{TBT} / \mathrm{mol} \mathrm{SA})^{0.5}$. Very interesting to note, that for PBSu an almost equal catalyst dependence (i.e. 0.51 ) was observed in the literature [3].

Table 2: $\quad$ Numerical values of the kinetic rate constants.

\begin{tabular}{lcccc}
\hline Polymer & Symbol & $\begin{array}{c}\text { Catalyst amount } \\
\left(\times 10^{4} \mathrm{~mol}\right. \\
\mathrm{TBT} / \mathrm{mol} \mathrm{SA})\end{array}$ & $\begin{array}{c}\mathrm{k}_{1}=\mathrm{k}_{2} \\
\left(\times 10^{3} \mathrm{~L} / \mathrm{mol} \cdot\right. \\
\mathrm{min})\end{array}$ & $\begin{array}{c}\mathrm{k}_{6} \\
\left(\times 10^{3} \mathrm{~L} / \mathrm{mol}\right. \\
\mathrm{min})\end{array}$ \\
\hline Poly(propylene succinate) & PPSu(H) & 1.5 & 1.5 & 1.0 \\
Poly(propylene succinate) $)$ & PPSu(T) & 3.0 & 2.2 & 0.8 \\
Poly(propylene succinate) & PPSu(D) & 6.0 & 3.0 & 0.7 \\
Poly(ethylene succinate) & PESu & 3.0 & 1.8 & 0.6 \\
Poly(butylene succinate) & PBSu & 3.0 & 2.7 & 1.1 \\
\hline
\end{tabular}

The effect of the type of glycol used (i.e. EG, PG or BG) on the esterification reaction is examined next. Results on the water conversion as a function of time appear in Figure 2. It is seen that use of BG leads to slightly higher reaction rates than PG, which in turn is also slightly faster compared to EG. Again the theoretical simulation model fits the experimental data very well.

From theoretical results, it was found that different glycols do not influence much the number average degree of polymerization of the oligomers produced during the esterification step. In contrast, these values were affected much from the amount of catalyst used. 


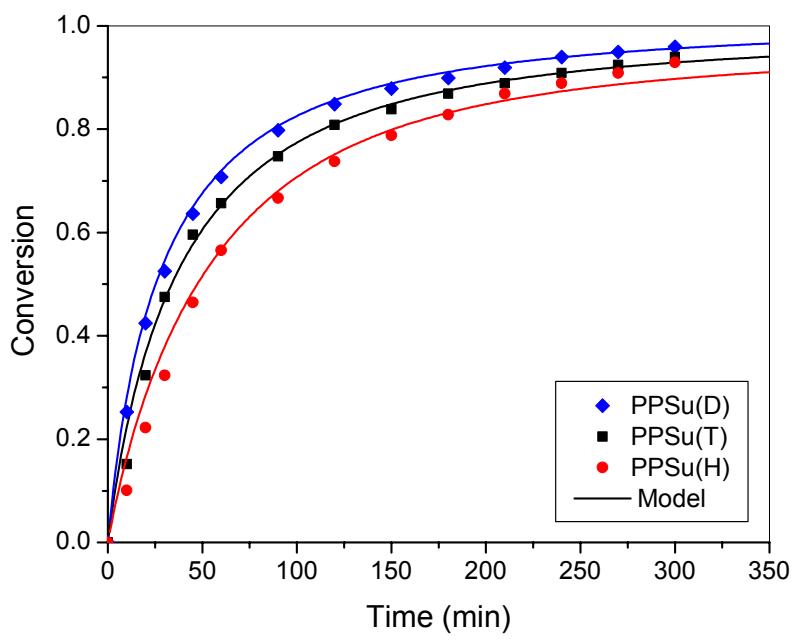

Figure 1: Conversion $\left(\mathrm{X}_{\mathrm{w}}\right)$ versus time for the esterification reaction of $\mathrm{PPSu}$ at $190^{\circ} \mathrm{C}$ with different amounts of added catalyst. Experimental data (discrete points) and theoretical model simulation results.

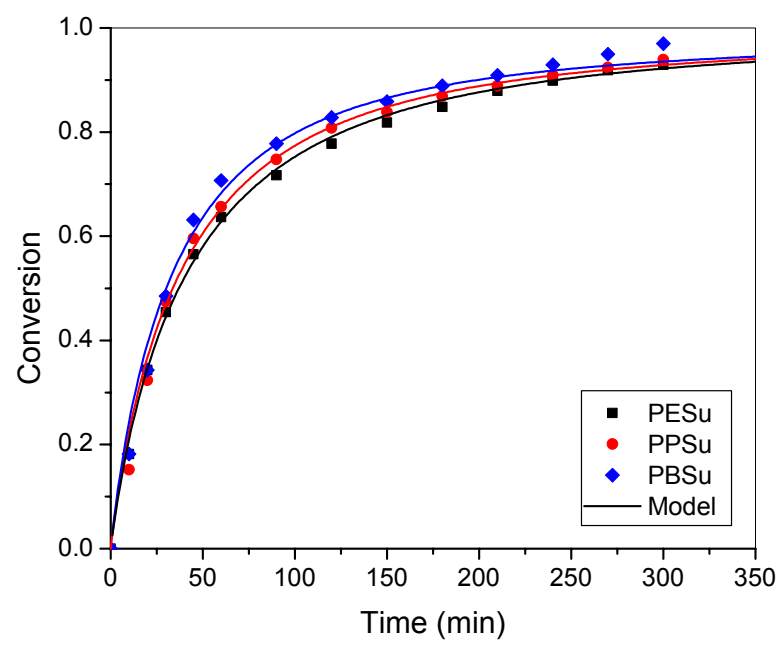

Figure 2: $\quad$ Conversion $\left(\mathrm{X}_{\mathrm{w}}\right)$ versus time for the esterification reaction of PESu, $\mathrm{PPSu}$ and PBSu at $190^{\circ} \mathrm{C}$ and $3 \times 10^{-4} \mathrm{~mol} \mathrm{TBT} / \mathrm{mol} \mathrm{SA}$. Experimental data (discrete points) and simulation results

\subsection{Biodegradation studies (kinetics of the enzymatic hydrolysis)}

The kinetics of enzymatic hydrolysis has been studied in literature for several polyesters $[4,5]$. The reaction temperature mainly affects hydrolysis rate and the 
enzyme used. For aliphatic polyesters, as the temperature increases, approximating melting temperature, enzymatic hydrolysis is accelerated [4]. Thus, quite satisfactory hydrolysis rates are obtained when the temperature is 10 $20^{\circ} \mathrm{C}$ lower than the polymers melting point. The most commonly used temperature for enzymatic hydrolysis studies is that of $37^{\circ} \mathrm{C}$. In this study, it was decided to study hydrolysis at $30^{\circ} \mathrm{C}$, since $\mathrm{PPSu}$ melts at a relatively low temperature $\left(\mathrm{T}_{\mathrm{m}}=44^{\circ} \mathrm{C}\right)$ and at higher temperatures it would be significantly softened.

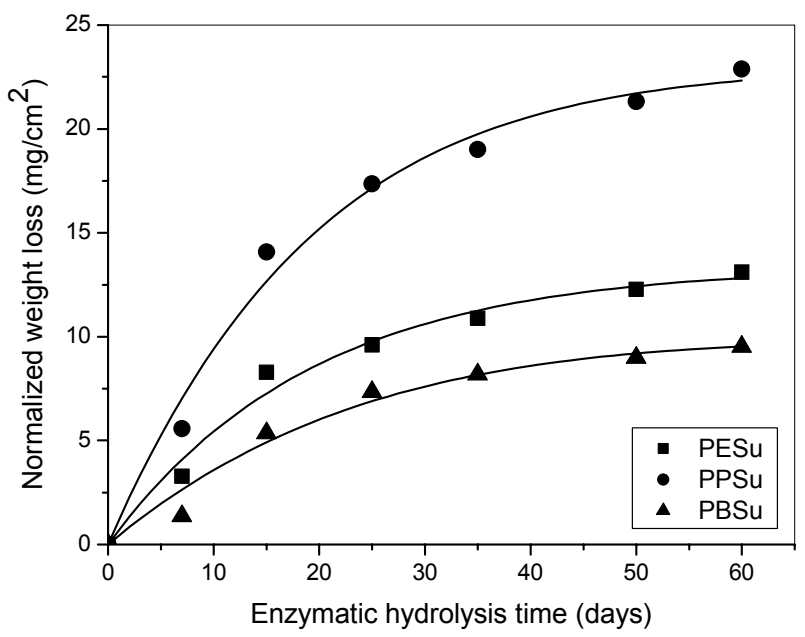

Figure 3: Weight loss of aliphatic polyester films during enzymatic hydrolysis. Curves through the experimental points represent the best fit of eqn (30).

As it is well known enzymatic hydrolysis is a heterogeneous process. Enzymes are attached on the surface of an insoluble substrate and hydrolysis takes place via surface erosion. In general, the internal parts of polyesters specimens are not attacked until extended holes are created onto surface allowing the enzymes to penetrate and attack to the main body. Thus, hydrolysis rates are decreased after consumption of the amorphous material of the surface. Afterwards, a layer of crystalline domains remains, where only slow degradation may occur. This inhibits penetration of water into the body of the film resulting in decreased rates of hydrolysis. In Figure 3 it was observed that PPSu exhibits the highest weight loss values, while PBSu showed the lowest ones. Since in our study the prepared polyesters have almost identical molecular weights, crystallinity seems to be the predominant factor that controls the biodegradation rates. It is therefore the higher degree of crystallinity of the PBSu samples, as was found by DSC and WAXD, [6] which resulted in lower degrees of biodegradation comparing to that of PESu or PPSu. PPSu, with the lower crystallinity, hydrolyses faster comparing to the other polyesters. 
In order to quantify these results a simple theoretical kinetic model is developed to predict the time evolution of the polymer weight loss. It should be pointed out that the derivation of a detailed mathematical model is rather complicated, since mass transfer phenomena (between the solid-polymer phase and the enzyme) occur in parallel to the chemical reaction. This model is formally based on the kinetic mechanism of enzymatic hydrolysis according to the Michaelis-Menten scheme:

$$
\begin{aligned}
& E+S \stackrel{k_{1}}{\underset{k_{-1}}{\rightleftarrows}} E S \\
& E S \stackrel{k_{2}}{\longrightarrow} P+E
\end{aligned}
$$

where $\mathrm{E}, \mathrm{S}$ and $\mathrm{P}$ represent the enzyme, substrate-polymer and hydrolysis reaction products, respectively and ES is the enzyme/substrate complex.

According to this kinetic scheme the mass balance of the substrate - polymer can be expressed from:

$$
\frac{d C_{S}}{d t}=-k_{1} C_{E} C_{S}+k_{-1} C_{E S}
$$

However, since reaction (24) involves a solid surface it is better to transform eqn (26) in surface area terms:

$$
\frac{d(A(1-\theta))}{d t}=-k_{1} C_{E} A(1-\theta)+k_{-1} A \theta \Rightarrow \frac{d(\theta)}{d t}=k_{1} C_{E}-\left(k_{1} C_{E}+k_{-1}\right) \theta
$$

where $A$ is the substrate surface area, $\theta$ is the fraction of substrate occupied by the ES complex, (1- $\theta)$ is the "free" surface fraction and $\mathrm{C}_{\mathrm{E}}$ is the enzyme concentration. The same terms have been also used in literature [5].

Assuming $\mathrm{C}_{\mathrm{E}}$ to be constant eqn (27), can be easily integrated to give:

$$
\theta=\frac{k_{1} C_{E}}{k_{1} C_{E}+k_{-1}}\left\{1-\exp \left[-\left(k_{1} C_{E}+k_{-1}\right) t\right]\right\}
$$

The experimentally measured normalized weight loss in $\mathrm{mg} / \mathrm{cm}^{2}, \mathrm{M}_{\mathrm{S} 0}-\mathrm{M}_{\mathrm{S}}$ can be correlated to $\theta$ according to the following equation:

$$
M_{S 0}-M_{S}=\frac{N_{S 0}}{A_{0}}-\frac{N_{S}}{A}
$$

where the symbol $\mathrm{N}$ is used to denote the substrate mass and the subscript 0 refers to initial conditions (i.e. time equal to zero).

Assuming that the substrate surface area, A, is proportional to the free surface fraction powered to a constant value, $\alpha$, according to $A=A_{0}(1-\theta)^{a}$, eqn (29) is expressed as:

$$
M_{S 0}-M_{S}=\frac{N_{S 0}}{A_{0}}\left[1-(1-\theta)^{1-a}\right]
$$

If a is set equal to zero a constant surface area during the reaction is assumed, furthermore, if $\alpha$ is set equal to 1 a constant normalized weight loss is obtained. In this study the arithmetic mean value was considered, i.e. $\alpha=0.5$.

Eqns (30) and (28) can then be used to fit the experimental data of the normalized substrate weight loss as a function of time for the three individual 
polymers used. In these equations three different fitting parameters were identified, i.e. the kinetic rate constants $\mathrm{k}_{1}, \mathrm{k}_{-1}$ and the initial substrate mass $\mathrm{N}_{\mathrm{S} 0} / \mathrm{A}_{0}$, which was kept as a fitting parameter in order to have an estimation of the initial effective polymer mass, which actually is biodegradable. A non-linear curve-fitting algorithm was used based on the Levenberg-Marquardt method and the best fitting values for the parameters are presented in Table 3.

Table 3: Kinetic and physical parameters determined by fitting eqn (30) to the experimental data of enzymatic hydrolysis of aliphatic polyesters.

\begin{tabular}{lccc}
\hline Polyester & $\begin{array}{c}\mathrm{k}_{1} \\
(\mathrm{~mL} / \mathrm{mg} \times \text { days })\end{array}$ & $\begin{array}{c}\mathrm{k}_{-1} \\
\left(\text { days }^{-1}\right)\end{array}$ & $\begin{array}{c}\mathrm{N}_{\mathrm{S}_{0}} / \mathrm{A}_{0} \\
\left(\mathrm{mg} / \mathrm{cm}^{2}\right)\end{array}$ \\
\hline PESu & 0.021 & 0.036 & 66 \\
PPSu & 0.032 & 0.028 & 72 \\
PBSu & 0.053 & 0.009 & 16 \\
\hline
\end{tabular}

As it can be seen going from PESu to PBSu an increase in the right direction kinetic rate constant was obtained with the reverse effect observed in the left direction rate constant. This means that as the spacing between ester groups increases the polymer is more susceptible to enzymatic attack. Furthermore, the best fitting value of the initial normalized substrate mass, $\mathrm{N}_{\mathrm{S} 0} / \mathrm{A}_{0}$, taking part in the enzymatic hydrolysis is larger for PPSu followed by PESu and PBSu. This result is in direct accordance with the polyester crystallinity data. As it was reported [6], PPSu exhibits the less degree of crystallinity followed by PESu and PBSu. Hence the assumption that the effective material mass, taking place in the degradation, is that of the amorphous polymer is confirmed.

\section{Acknowledgement}

This work was funded by the E.K.T. / Е.П.Е.A.E.K. II in the framework of the research program PYTHAGORAS II, Metro 2.6.

\section{References}

[1] Karayannidis, G.P., Roupakias, C.P., Bikiaris, D.N. \& Achilias, D.S., Polymer, 44, 931, 2003.

[2] Kang, C.K., Lee, B.C. \& Ihm, D.W., Journal of Applied Polymer Science, 60, 2007, 1996.

[3] Park, S.S., Jun, H.W. \& Im, S.S., Polymer Engineering \& Science, 38(6), 905, 1998.

[4] Marten, E., Müller, R.-J. \& Deckwer, W.-D., Polymer Degradation \& Stability, 80, 485, 2003.

[5] Scandola, M., Focarete, M.L. \& Frisoni, G., Macromolecules, 31, 3846, 1998.

[6] Bikiaris, D.N., Papageorgiou, G.Z. \& Achilias, D.S., Polymer Degradation \& Stability, 80, 485, 2003. 